MZ-TH/01-31

hep-ph/0111206

November 2001

\title{
An interpolation of the vacuum polarization function for the evaluation of hadronic contributions to the muon anomalous magnetic moment
}

\author{
S. Groote, J.G. Körner and A.A. Pivovarov \\ Institut für Physik, Johannes-Gutenberg-Universität, \\ Staudinger Weg 7, D-55099 Mainz, Germany \\ and \\ Institute for Nuclear Research of the \\ Russian Academy of Sciences, Moscow 117312, Russia
}

\begin{abstract}
We propose a simple parameterization of the two-point correlator of hadronic electromagnetic currents for the evaluation of the hadronic contributions to the muon anomalous magnetic moment. The parameterization is explicitly done in the Euclidean domain. The model function contains a phenomenological parameter which provides an infrared cutoff to guarantee the smooth behavior of the correlator at the origin in accordance with experimental data in $e^{+} e^{-}$annihilation. After fixing a numerical value for this parameter from the leading order hadronic contribution to the muon anomalous magnetic moment the next-to-leading order results related to the vacuum polarization function are accurately reproduced. The properties of the four-point correlator of hadronic electromagnetic currents as for instance the so-called light-by-light scattering amplitude relevant for the calculation of the muon anomalous magnetic moment are briefly discussed.
\end{abstract}




\section{Introduction}

The description of strong interactions based on QCD proves to be very successful for processes at large energies where the coupling constant is small due to the property of asymptotic freedom [1]. This makes perturbation theory (PT) computations reliable. At low energies the problem of strong coupling prevents using QCD as an unambiguous theoretical tool for computations of physical observables and various phenomenological models are introduced. These models are inspired by QCD but it is difficult to establish a quantitative relation between the underlying theory and a model used in practice. For instance, the chiral perturbation theory (ChPT) for Goldstone modes is a very general model in the sense that this effective theory provides the expansion of the full QCD amplitudes at low energies according to symmetry principles without using special assumptions. In this respect the amplitudes of ChPT represent the low energy limits of the exact QCD amplitudes based on current algebra and collect the current algebra results in a compact way [2]. Of course, for the applicability of ChPT one should guarantee that the expansion in the energy is well convergent (at least explicitly). ChPT is very practical in describing interactions of pions (as lightest hadrons) with nucleons or resonances at low energy in the small momentum (and mass) expansion [3, 此. Thus the description of strong interactions at low energies relies on phenomenological models with explicit introduction of elementary hadron fields or on the closely related approaches based on general principles of analyticity, unitarity and symmetry [5]. A general idea of linking this approach for the description of hadrons at low energies with QCD is the concept of duality which means that the description of inclusive observables which are sensitive to the contribution of many particles is simpler than that of exclusive processes and can be represented by almost free fermions or weakly coupled quarks [6]. This concept works well for infrared (IR) soft observables in $\tau$-decays and other sum rules where the limit of massless quarks is nonsingular [7, 8, 9, 10, 11, 12]. For the IR sensitive observables the realization of the duality concept for the light modes is not quite straightforward since the IR cutoff explicitly enters the calculation. In such cases the IR cutoff is usually taken from experiment such as the mass of a real hadron.

Strong interactions at low energies play an important role in precision tests of the Standard Model as a whole. They are involved in the evaluation of the CP-violating structure in the electroweak sector through hadronic matrix elements, the computation of which constitutes a main obstacle for the progress of a quantitative analysis of the nonleptonic kaon decays relevant for determination of the quark mixing matrix [13, 14 and mixing of neutral pseudoscalar states [15]. In other tests of the Standard Model strong interactions enter as small corrections to very accurately measured observables. Examples of high precision observables of the Standard Model with important contributions from strong interactions (as corrections to the leading leptonic effects) are the running electromagnetic (EM) coupling constant at the scale of the $Z$-boson mass and the muon anomalous magnetic moment (MAMM). The numerical values of these quantities put some constraints on the Standard Model parameters and can also serve as triggers of new physics beyond the Standard Model.

A numerical value of the muon anomalous magnetic moment (MAMM) is measured experimentally with high precision [16, 17]. The value presented in a recent review [18] reads

$$
a_{\mu}^{\exp }=116592023(151) \times 10^{-11}
$$

with an uncertainty of $151 \times 10^{-11}$. In future experiments a goal is set to reach the accuracy of $40 \times 10^{-11}$. In theoretical computations the leading contribution to the MAMM is given by

$$
a_{\mu}^{\mathrm{Schw}}=\frac{\alpha}{2 \pi}
$$


(first calculated by Schwinger) where $\alpha$ is the fine structure constant $\alpha^{-1}=137.036 \ldots$ [16]. The MAMM is sensitive to the IR region of integration in perturbation theory diagrams. For the leptonic (QED) part of the contribution this is reflected in a strong dependence on the electron mass. Theoretically the purely leptonic part is computed in perturbative QED with finite lepton masses which leads to a function $A\left(m_{e} / m_{\mu}, m_{\mu} / m_{\tau}\right)$ which is known analytically to three loops. As $m_{e} \ll m_{\mu} \ll m_{\tau}$ the ratios $m_{e} / m_{\mu}$ and $m_{\mu} / m_{\tau}$ are small and the function $A\left(m_{e} / m_{\mu}, m_{\mu} / m_{\tau}\right)$ can be expanded in these ratios to simplify calculations. The contribution of the muon leads to diagrams with a single scale $m_{\mu}$ that makes it simpler to compute. The complete analytical calculation is technically very complicated already at the level of three loops [19]. The nontrivial diagrams in higher orders were computed numerically [20, 21]. The present value of the QED contribution to the muon anomalous magnetic moment reads 222, 23] (as a review see [18])

$$
a_{\mu}^{\mathrm{QED}}=116584705.7(2.9) \times 10^{-11} .
$$

Computation of the electroweak (EW) corrections to the MAMM has also been performed in perturbation theory [24]. The EW contribution is now known with two-loop accuracy (as a review see [18]),

$$
a_{\mu}^{\mathrm{EW}}=152(4) \times 10^{-11} .
$$

The hadronic contribution to the MAMM is sensitive to the infrared region and cannot be computed in perturbative QCD with light quarks. The current masses of light quarks are too small to provide a necessary infrared cutoff and explicit models of hadronization are required for a quantitative analysis [25, 26, 27]. The hadronic contribution is the main uncertainty in the theoretical computation of the MAMM in the Standard Model. Assuming the validity of the Standard Model for the description of elementary particle interactions

$$
a_{\mu}^{\exp }=a_{\mu}^{\mathrm{SM}},
$$

the numerical value for the hadronic contribution to the MAMM in the Standard Model is given by

$$
\left.a_{\mu}^{\mathrm{had}}\right|_{\mathrm{as}}=a_{\mu}^{\exp }-a_{\mu}^{\mathrm{QED}}-a_{\mu}^{\mathrm{EW}}=\left(7165.3 \pm\left. 151\right|_{\exp } \pm\left. 2.9\right|_{\mathrm{QED}} \pm\left. 4\right|_{\mathrm{EW}}\right) \times 10^{-11} .
$$

The experimental error dominates the uncertainty.

Since the hadronic contribution is sensitive to the details of the strong coupling regime of QCD at low energies and cannot be unambiguously computed in a perturbation theory framework the theoretical prediction for the MAMM in the Standard Model depends crucially on how this contribution is estimated [28]. In the absence of a reliable theoretical tool for the computation in this region one turns to experimental data on low-energy hadron interactions for extracting a numerical value [29]. In general terms the hadronic contribution to the MAMM is determined by the correlation functions of electromagnetic (EM) currents. Since a source for the EM current is readily available for a wide range of energies, one tries to extract these functions or some of their characteristics relevant for a particular application from experiment. Without explicit use of QCD the correction $a_{\mu}^{\text {had }}$ in the Standard Model is generated through the EM interaction $e j_{\mu}^{\text {had }} A^{\mu}$ with $j_{\mu}^{\text {had }}$ being the hadronic part of the EM current. At the leading order ( $\alpha^{2}$ in formal power-counting) only the two-point correlation function of the EM currents emerges in the analysis of hadronic contributions to the MAMM. At the next-to-leading order $\left(\alpha^{3}\right)$ a four-point correlation function appears. These correlators are not calculable perturbatively in the region essential for the determination of the hadronic contributions to the MAMM. The leading 


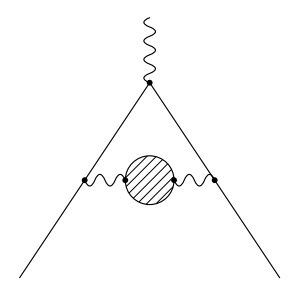

Figure 1: The leading order hadronic contribution to the MAMM, the shaded bubble indicates the hadronic two-point correlator

contribution to the MAMM comes from the two-point correlator referred to as the hadronic part of the photon vacuum polarization contribution while the four-point function first emerges at the $\alpha^{3}$ order, most explicitly as the light-by-light scattering amplitude. To avoid using QCD in the strong coupling mode one can extract the necessary contribution to the MAMM by studying these two correlation

functions experimentally without an explicit realization of the hadronic EM current $j_{\mu}^{\text {had }}$ in terms of elementary fields. Another possibility which is close in spirit is to use phenomenological models to saturate these correlators with contributions of real hadrons at low energies [22, 30, 31, 32, 33]. There is also a possibility to use a concept of duality between hadron and quark-gluon descriptions modified for handling IR sensitive observables [34, 35]. In the following we discuss this last option.

\section{Hadronic contribution at leading order}

At the leading order in $\alpha$ the hadronic contribution is described by the correlator

$$
i \int\left\langle T j_{\mu}^{h a d}(x) j_{\nu}^{h a d}(0)\right\rangle e^{i q x} d x=\left(q_{\mu} q_{\nu}-g_{\mu \nu} q^{2}\right) \Pi^{\text {had }}\left(q^{2}\right)
$$

in terms of a single function $\Pi^{\text {had }}\left(q^{2}\right)$ of one variable $q^{2}$. The contribution of $\Pi^{\text {had }}\left(q^{2}\right)$ to the muon anomalous magnetic moment (e.g. [37]) is given by

$$
a_{\mu}^{\mathrm{had}}(\mathrm{LO})=4 \pi\left(\frac{\alpha}{\pi}\right)^{2} \int_{4 m_{\pi}^{2}}^{\infty} \frac{d s}{s} K(s) \operatorname{Im} \Pi^{\mathrm{had}}(s)
$$

with a one-loop kernel of the form

$$
K(s)=\int_{0}^{1} d x \frac{x^{2}(1-x)}{x^{2}+(1-x) s / m^{2}} .
$$

Here $\operatorname{Im} \Pi^{\text {had }}(s)=\operatorname{Im}\left\{\left.\Pi^{\text {had }}\left(q^{2}\right)\right|_{q^{2}=s+i 0}\right\}, m$ is a muon mass.

The leading order hadronic contribution to the MAMM as depicted in Fig. 1 is represented by an integral over the hadron spectrum and no specific information about the function $\operatorname{Im} \Pi^{\text {had }}(s)$ is necessary point-wise. However, a QCD approach based on light quark duality in the massless approximation is not directly applicable as the integral in Eq. (8) is IR sensitive and depends strongly on the threshold structure of the function $\Pi^{\text {had }}\left(q^{2}\right)$. In most applications the threshold structure is extracted from experiment. To the leading order in $\alpha$ the function $\operatorname{Im} \Pi^{\text {had }}(s)$ can uniquely be identified with data from $e^{+} e^{-}$annihilation into hadrons. Introducing the experimental $R^{\exp }(s)$ ratio

$$
R^{\exp }(s)=\frac{\sigma\left(e^{+} e^{-} \rightarrow \text { hadrons }\right)}{\sigma\left(e^{+} e^{-} \rightarrow \mu^{+} \mu^{-}\right)}, \quad s=\left(p_{e^{+}}+p_{e^{-}}\right)^{2}
$$




\begin{tabular}{|r|l|l|l|l|}
\hline reference & $a_{\mu}^{\text {had }}(\mathrm{LO})$ & $a_{\mu}^{\text {had }}(\mathrm{LO} ;$ light $)$ & $m_{\mathrm{eff}}[\mathrm{MeV}]$ & $m_{q}[\mathrm{MeV}]$ \\
\hline Ref. [28] & $7011(94) \times 10^{-11}$ & $6940(96) \times 10^{-11}$ & $200.5(1.6)$ & $178.7(1.4)$ \\
Ref. [36] & $7024(154) \times 10^{-11}$ & $6953(155) \times 10^{-11}$ & $200.3(2.5)$ & $178.5(2.2)$ \\
Ref. [38] & $6950(150) \times 10^{-11}$ & $6879(151) \times 10^{-11}$ & $201.5(2.5)$ & $179.6(2.2)$ \\
Ref. [39] & $6924(62) \times 10^{-11}$ & $6853(65) \times 10^{-11}$ & $201.9(1.1)$ & $180.0(1.0)$ \\
Ref. [29] & $6988(111) \times 10^{-11}$ & $6917(112) \times 10^{-11}$ & $200.9(1.8)$ & $179.0(1.6)$ \\
\hline optimistic & $6961(43) \times 10^{-11}$ & $6892(44) \times 10^{-11}$ & $201.3(0.7)$ & $179.4(0.6)$ \\
conservative & $6979(114) \times 10^{-11}$ & $6908(116) \times 10^{-11}$ & $201.0(1.9)$ & $179.2(1.7)$ \\
\hline
\end{tabular}

Table 1: Comparison of a selection of different determinations of the leading order contribution to $a_{\mu}^{\text {had }}$ in the literature. For the first five lines the first column indicates the reference, the second column the cited value. In the third column the leading order contributions from heavy quarks are subtracted. The sixth and seventh line show the optimistic and conservative estimate for the mean value. The fourth and fifth column list the resulting values for $m_{\mathrm{eff}}$ and $m_{q}$ for model 1 and model 2 , resp.

and identifying it with the theoretical quantity $12 \pi \operatorname{Im} \Pi^{\text {had }}(s)$ one finds

$$
a_{\mu}^{\text {had }}(\mathrm{LO})=\frac{1}{3}\left(\frac{\alpha}{\pi}\right)^{2} \int_{4 m_{\pi}^{2}}^{\infty} \frac{R^{\exp }(s) K(s)}{s} d s .
$$

The contribution to the MAMM based on the representation given in Eq. (11) is well studied. Several recent determinations based on a thorough treatment of various sets of data are given in the second column of Table 1. All values given in Table 1 are consistent within errors. We can take only a small selection of results because other results are again within errors but with mean values slightly different. For the same reason, the old historical result given in Ref. [28] could be cited as well. Table 1 gives two different mean values, namely an optimistic and a conservative average. The optimistic average uses the error estimates of the different results as weights, assuming that the results are independent. Here we use the method presented in Sec. 4.3 of Ref. [36]. But because all treatments in the literature are mainly based on the close data sets taken for the analysis, this assumption might not be true. Therefore, we also give a conservative average which reads

$$
a_{\mu}^{\text {had }}(\mathrm{LO})=6979(114) \times 10^{-11} .
$$

This average is in agreement with the experimental result in Eq. (6) within error bars. The statistical correlation of errors coming from the experimental value in Eq. (1) and the leading order hadronic data in Eq. (12) is supposed to be small as they are determined by different sources. Other errors are negligible. For the target experimental error of the MAMM at the level of $40 \times 10^{-11}$ [22] the value in Eq. (12) is somewhat small and NLO hadronic corrections should be taken into account. In order to obtain a naive order-of-magnitude estimate for this contribution, we take the value of the leading order suppressed by $\alpha \approx 1 / 137$ which gives roughly $50 \times 10^{-11}$. Writing

$$
\left.a_{\mu}^{\text {had }}\right|_{\text {th }}=a_{\mu}^{\text {had }}(\mathrm{LO})+a_{\mu}^{\text {had }}(\mathrm{NLO})
$$

and comparing with Eq. (6) one has (in units of $10^{-11}$ )

$$
\begin{aligned}
a_{\mu}^{\mathrm{had}}(\mathrm{NLO}) & =7165 \pm\left. 151\right|_{\mathrm{exp}} \pm\left. 2.9\right|_{\mathrm{QED}} \pm\left. 4\right|_{\mathrm{EW}}-\left.6979(114)\right|_{\mathrm{LO}} \\
& =186 \pm\left. 151\right|_{\mathrm{exp}} \pm\left. 2.9\right|_{\mathrm{QED}} \pm\left. 4\right|_{\mathrm{EW}} \pm\left. 114\right|_{\mathrm{had}} .
\end{aligned}
$$


Assuming the statistical independence of the uncertainties one finds after adding them in quadratures

$$
a_{\mu}^{\text {had }}(\mathrm{NLO})=(186 \pm 189) \times 10^{-11}
$$

which would not allow one to see higher order hadronic effects clearly. Assuming that the mean value of $a_{\mu}^{\exp }$ in the planned experiment will not change and the target accuracy $40 \times 10^{-11}$ will be reached, one finds a numerical value for the NLO hadronic contribution of

$$
a_{\mu}^{\text {had }}(\mathrm{NLO})=(186 \pm 121) \times 10^{-11}
$$

which makes the NLO hadronic effects noticeable at the level of two standard deviations. If the mean value of $a_{\mu}^{\exp }$ will change in the range of the present experimental uncertainty of $151 \times 10^{-11}$, the NLO hadronic effects can be more or less pronounced.

To create a framework for the analysis of hadronic contributions at NLO based on duality arguments we rewrite the LO expression for the hadronic contribution to the MAMM given in Eq. (8) in a different form. From general principles a two-point correlator $\Pi\left(q^{2}\right)$ as a function of the complex variable $q^{2}$ can have a cut along the positive semiaxis $s>0$ with a positive discontinuity [40]. This spectral condition plays a crucial role in the analysis of the structure of the two-point correlators and related observables [41, 42]. The dispersion relation reads

$$
\Pi\left(q^{2}\right)=\frac{1}{\pi} \int_{0}^{\infty} \frac{d s}{s-q^{2}} \operatorname{Im} \Pi(s)-\text { subtractions. }
$$

For massive pions the experimental spectrum in $e^{+} e^{-}$annihilation starts from $4 m_{\pi}^{2}$ and the subtraction at the origin is possible as $\operatorname{Im} \Pi^{\text {had }}(s)=0$ for $s<4 m_{\pi}^{2}$. The dispersion representation with subtraction at the origin reads

$$
\Pi^{\text {had }}\left(q^{2}\right)=\frac{q^{2}}{\pi} \int_{4 m_{\pi}^{2}}^{\infty} \frac{d s}{s} \frac{\operatorname{Im} \Pi^{\mathrm{had}}(s)}{s-q^{2}}
$$

which implies the normalization condition $\Pi^{\text {had }}(0)=0$. Using Eqs. (16, 17) and Eqs. (8,9) one can rewrite the LO contribution to the MAMM as an integral over Euclidean values of $q^{2}$ for $\Pi^{\text {had }}\left(q^{2}\right)$,

$$
a_{\mu}^{\text {had }}(\mathrm{LO})=4 \pi^{2}\left(\frac{\alpha}{\pi}\right)^{2} \int_{0}^{\infty}\left\{-\Pi^{\text {had }}(-t)\right\} W(t) d t
$$

with

$$
W(t)=\frac{4 m^{4}}{\sqrt{t^{2}+4 m^{2} t}\left(t+2 m^{2}+\sqrt{t^{2}+4 m^{2} t}\right)^{2}} .
$$

Such a representation is well known and is often written as a parametric integral [43, 44].

The representation in Eq. (11) is suitable for the evaluation of the hadronic contributions to the MAMM by using experimental data, since it can be rewritten in terms of the hadronic cross section for $e^{+} e^{-}$annihilation. The representation in Eq. (18) is more suitable for a theoretical study as perturbation theory should preferably be applied in the Euclidean domain. Integration by parts in Eq. (18) gives

$$
\frac{1}{\pi} \int_{4 m_{\pi}^{2}}^{\infty} \frac{d s}{s} K(s) \operatorname{Im} \Pi^{\mathrm{had}}(s)=\int_{0}^{\infty}\left(-\frac{d \Pi^{\mathrm{had}}(-t)}{d t}\right) F(t) d t, \quad F(t)=\int_{t}^{\infty} W(\zeta) d \zeta
$$




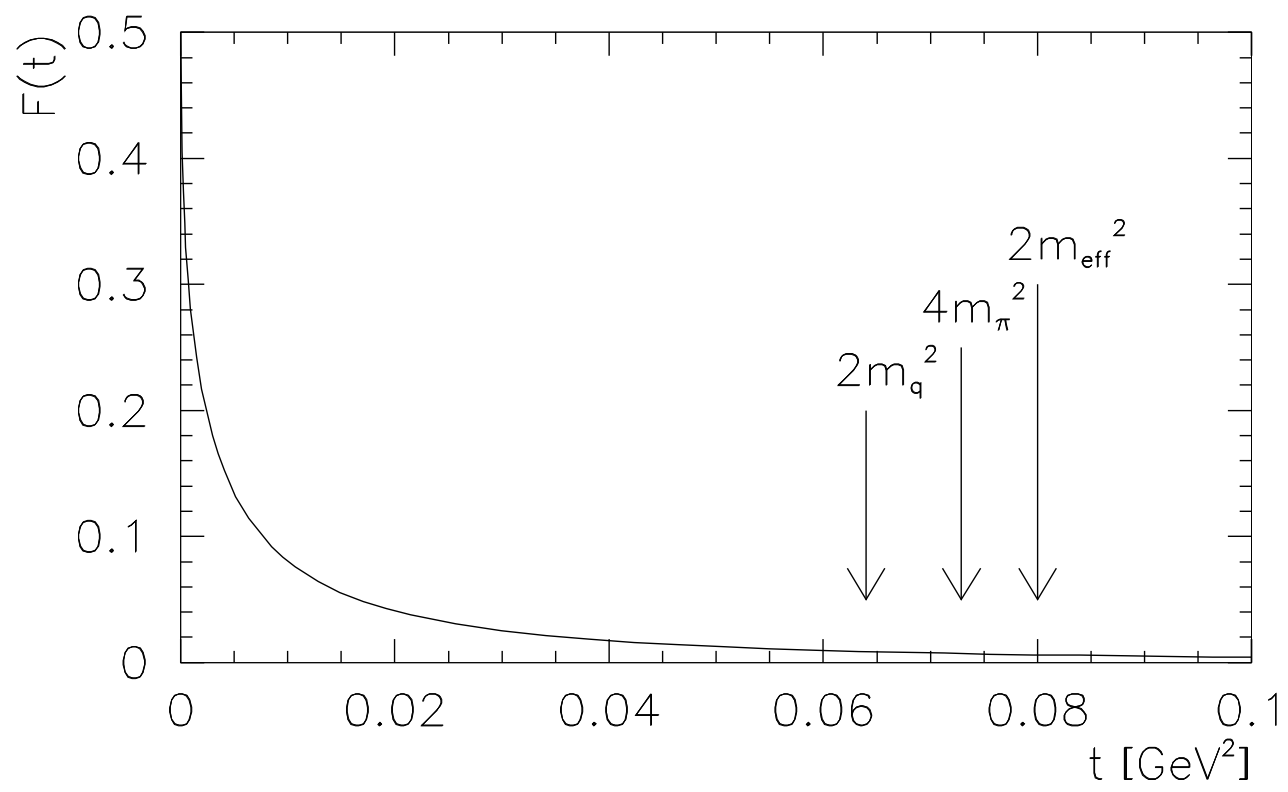

Figure 2: The LO Euclidean weight function $F(t)$

with

$$
F(t)=\frac{1}{2}\left(\frac{t+2 m^{2}-\sqrt{t^{2}+4 m^{2} t}}{t+2 m^{2}+\sqrt{t^{2}+4 m^{2} t}}\right)=\frac{2 m^{4}}{\left(t+2 m^{2}+\sqrt{t^{2}+4 m^{2} t}\right)^{2}} .
$$

The behavior of the function $F(t)$ is shown in Fig. 2 for small and large $t$. It reads

$$
\left.F(t)\right|_{t \rightarrow 0}=\frac{1}{2}-\frac{\sqrt{t}}{m}+O(t),\left.\quad F(t)\right|_{t \rightarrow \infty}=\frac{m^{4}}{2 t^{2}}+O\left(1 / t^{3}\right) .
$$

The surface terms of the integration by parts vanish because the integrand in Eq. (18) satisfies the conditions $\left|\Pi^{\text {had }}(-t)\right|<C \sqrt{t}$ at small $t$ and $\left|\Pi^{\text {had }}(-t)\right|<C^{\prime} t^{-2}$ at large $t$ with some given constants $C, C^{\prime}$.

A key physical quantity of the analysis is the derivative of the hadron vacuum polarization function $d \Pi^{\text {had }}(-t) / d t$ which is closely related to the Adler function

$$
D(t)=-t \frac{d \Pi^{\mathrm{had}}(-t)}{d t} .
$$

The quantity $d \Pi^{\text {had }}(-t) / d t$ can be computed in perturbative QCD with massless quarks for large $t$,

$$
-\frac{d \Pi^{\mathrm{had}}(-t)}{d t}=\frac{e_{q}^{2} N_{c}}{12 \pi^{2} t}\left(1+\frac{\alpha_{s}(t)}{\pi}\right)
$$

where $e_{q}$ is the charge of the quark in units of the elementary (electron) electric charge and $N_{c}$ is the number of colors. Computation at small $t$ in perturbation theory is not possible for light quarks with small masses as the theory enters the regime of strong coupling. The behavior of the function $d \Pi^{\text {had }}(-t) / d t$ for small $t$ can be extracted from experiment where the lower limit of the spectrum is determined by the finite pion masses. This leads to a finite value for the function $d \Pi^{\text {had }}(-t) / d t$ at 
$t=0$. Using the patterns of small and large $t$ behavior of the function $d \Pi^{\text {had }}(-t) / d t$ for the light modes, we suggest an interpolation function $f(t)$ valid for all $t$ in the form

$$
-\frac{d \Pi^{\mathrm{had}}(-t)}{d t}=\frac{e_{q}^{2} N_{c}}{12 \pi^{2}} f(t), \quad f(t)=\frac{1}{t+\Delta} .
$$

Writing

$$
f(t)=-\frac{d p(t)}{d t}
$$

one has

$$
p(t)=\ln \left(\frac{\Delta}{t+\Delta}\right), \quad p(0)=0 .
$$

The analytic properties of the function $p(t)$ are given by the cut along the positive semiaxis starting at $s=\Delta$. The discontinuity across the cut is equal to one,

$$
r(s)=\frac{1}{\pi} \operatorname{Im} p(-s-i 0)=\theta(s-\Delta) .
$$

Thus the contribution to the MAMM contains an integral

$$
I(\Delta)=\int_{0}^{\infty} f(t) F(t) d t
$$

which is the basic quantity for the theoretical analysis. The analytical expression for $I(\Delta)$ is available but too cumbersome to be presented here. This expression is used in numerical calculations. However, in order to understand the integral in Eq. (29) more deeply, in particular, to find where the integral in Eq. (29) is saturated or what region of integration is important, an approximation can be useful and worth mentioning. The constant approximation for the function $f(t)$,

$$
f^{\operatorname{appr}}(t)=\text { const }=f(0)=\frac{1}{\Delta}
$$

gives

$$
I^{\operatorname{appr}}(\Delta)=f(0) \int_{0}^{\infty} F(t) d t=f(0) \frac{m^{2}}{3}=\frac{m^{2}}{3 \Delta} .
$$

This result represents the leading term of the series expansion of $I(\Delta)$ for small $m^{2}$. The series expansion of $I(\Delta)$ for small $m^{2}$ up to terms of order $m^{6}$ is given by

$$
I(\Delta)=\frac{1}{3} v+\left(\frac{19}{24}+\frac{1}{2} \ln v\right) v^{2}+\left(\frac{77}{30}+2 \ln v\right) v^{3}+\ldots
$$

with $v=m^{2} / \Delta$. This series converges nicely for small values of $v$.

The interpolation is only necessary for the light modes since the small $t$ behavior is nonperturbative. Heavy quarks can be treated in perturbation theory as their masses are rather large [16, 45, 46]. The contribution of $c$ and $b$ quarks to the MAMM reads [35]

$$
a_{\mu}^{\text {had }}(\mathrm{LO} ; \text { heavy })=71(18) \times 10^{-11}
$$

where we used $m_{c}=(1.6 \pm 0.2) \mathrm{GeV}$ and $m_{b}=(4.8 \pm 0.2) \mathrm{GeV}$. The uncertainty mainly results from the uncertainty in the $c$ quark mass. Using this result, the light mode contribution becomes

$$
a_{\mu}^{\text {had }}(\mathrm{LO} ; \text { light })=a_{\mu}^{\text {had }}(\mathrm{LO})-a_{\mu}^{\text {had }}(\mathrm{LO} ; \text { heavy })=6908(116) \times 10^{-11} .
$$



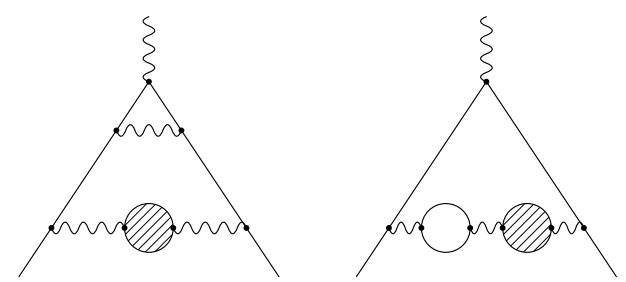

Figure 3: NLO contributions to the MAMM involving the contribution of the hadronic two-point correlator (left) and a lepton-hadron type (so-called double bubble) diagram (right)

The values for the different cited values are shown in the third column of Table 1. Using this experimental result one finds a numerical value for the IR parameter $\Delta$ of the interpolation function $f(t)$. Writing $\Delta=4 m_{\text {eff }}^{2}$ one obtains

$$
m_{\mathrm{eff}}=201.0 \pm 1.9 \mathrm{MeV} .
$$

The individual results are shown in the fourth column of Table 1. The function $r(s)$ of Eq. (28) is depicted in Fig. 5 for $m_{\mathrm{eff}}=201 \mathrm{MeV}$. This completes the quantitative description of the interpolation function for the two-point correlator of the light modes which can be used for the computation of the hadronic contributions at NLO. We cite this interpolation as model 1 in the following.

\section{Hadronic contribution at next-to-leading order}

The interpolation given by the function $f(t)$ for the two-point correlator with the numerical value of the phenomenological parameter from Eq. (35) is now used at NLO. Two of the NLO diagrams involving the hadronic two-point correlator are shown in Fig. 3. The NLO contribution is an integral of $\operatorname{Im} \Pi^{\text {had }}(s)$ with the two-loop kernel $K^{(2)}(s)$,

$$
a_{\mu}^{\mathrm{had}}(\mathrm{NLO})=4 \pi\left(\frac{\alpha}{\pi}\right)^{3} \int_{0}^{\infty} \frac{d s}{s} K^{(2)}(s) \operatorname{Im} \Pi^{\mathrm{had}}(s) .
$$

The analytical expression for the kernel $K^{(2)}(s)$ is known [47].

Assuming that the IR scale $M_{h}$ of the hadronic spectrum $\operatorname{Im} \Pi^{\text {had }}(s)$ is larger than $m$ one can use an expansion of $K^{(2)}(s)$ in $\mathrm{m}^{2} / \mathrm{s}$ under the integration sign in Eq. (36) to generate an expansion in $m / M_{h}$ for the integral. The IR scale of the data is set by the explicit cutoff at $\sqrt{s}=2 m_{\pi}$. Then one generates an expansion of $a_{\mu}^{\text {had }}(\mathrm{NLO})$ in $m^{2} / m_{\pi}^{2}$ using the data. The data-based analysis for the NLO effects of the vacuum polarization type [48] gives

$$
a_{\mu}^{\text {had }}(\operatorname{vac} ; \mathrm{NLO})=-101(6) \times 10^{-11} .
$$

In the proposed model the analysis is based on the explicit expression for the hadronic two-point correlator given in Eq. (25). In the model the hadronic scale is given by $\sqrt{\Delta}=2 m_{\text {eff }}$ and the expansion of the kernel results in the expansion of the integral in the variable $m^{2} / \Delta$ or $m^{2} / m_{\text {eff }}^{2}$. Note that convergence is not fast for the hadronic scale given by $m_{\pi}$ or $m_{\text {eff }}$. The integral in Eq. (36) can be rewritten in the Euclidean domain for $\Pi^{\text {had }}\left(q^{2}\right)$ in analogy to the LO treatment. For our purposes it suffices to use the expansion in $\mathrm{m}^{2} / \mathrm{s}$. We present some contributions separately for a comparison with the results from Ref. 48. 
The vertex part of the kernel has an expansion [48]

$$
\begin{gathered}
K_{\mathrm{ver}}^{(2)}(s)=2 \frac{m^{2}}{s}\left\{\left(\frac{223}{54}-\frac{\pi^{2}}{3}-\frac{23}{36} \ln \left(\frac{s}{m^{2}}\right)\right)\right. \\
+\frac{m^{2}}{s}\left(\frac{8785}{1152}-\frac{37 \pi^{2}}{48}-\frac{367}{216} \ln \left(\frac{s}{m^{2}}\right)+\frac{19}{144} \ln ^{2}\left(\frac{s}{m^{2}}\right)\right) \\
\left.+\frac{m^{4}}{s^{2}}\left(\frac{13072841}{432000}-\frac{883 \pi^{2}}{240}-\frac{10079}{3600} \ln \left(\frac{s}{m^{2}}\right)+\frac{141}{80} \ln ^{2}\left(\frac{s}{m^{2}}\right)\right)+\ldots\right\} .
\end{gathered}
$$

Generally, the terms of the expansion contain powers and logarithms of the variable $\mathrm{m}^{2} / \mathrm{s}$. For pure powers one can use a generating integral representation with a polynomial $P(x)$

$$
m^{2} \int_{0}^{1} \frac{d x P(x)}{m^{2} x+s}=\frac{m^{2}}{s} \sum_{n} a_{n}\left(\frac{m^{2}}{s}\right)^{n}, \quad a_{n}=\int_{0}^{1} d x P(x)(-x)^{n} .
$$

A given polynomial $P(x)$ restores the pure power expansion of Eq. (38). For the logarithmic part the generating integral representation can be chosen with a polynomial $G(x)$ of the form

$$
m^{2} \int_{0}^{1} \frac{d x G(x)}{s x+m^{2}}=G_{1}\left(m^{2} / s\right)+G_{2}\left(m^{2} / s\right) \ln \left(\frac{s}{m^{2}}\right) .
$$

The polynomial $G(x)$ generates polynomials $G_{1}(x), G_{2}(x)$ through Eq. (40). The mixture of pure powers due to the polynomial $G_{1}(x)$ leads to a redefinition of the polynomial $P(x)$ in Eq. (39). Using Eqs. (36, 39) one finds the expression for the pure power part of the expansion

$$
\left.\frac{1}{\pi} \int_{0}^{\infty} \frac{d s}{s} K^{(2)}(s)\right|_{\text {power }} \operatorname{Im} \Pi^{\text {had }}(s)=\int_{0}^{1} \frac{d x}{x} P(x)\left[-\Pi^{\text {had }}\left(-m^{2} x\right)\right]
$$

which reduces to derivatives of $\Pi^{\text {had }}(t)$ at the origin and gives the analytic part of the expansion in $m / M_{h}$. For the logarithmic part one finds the representation

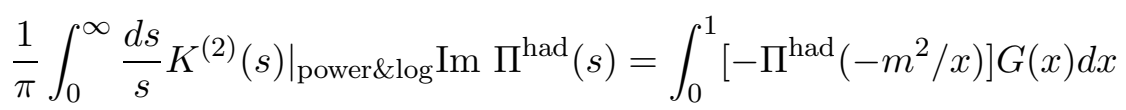

which is sensitive to the entire Euclidean domain and gives the nonanalytic part of the expansion containing $\ln \left(m / M_{h}\right)$. This procedure can be performed up to any finite order in $m^{2}$ and the whole calculation can be organized in a way such that only Euclidean values of momenta are necessary for $\Pi^{\text {had }}\left(q^{2}\right)$. Derivatives of the function $\Pi^{\text {had }}(t)$ at the origin emerging from Eq. (41) depend on the hadronic scale $M_{h}$ while the muon mass enters polynomially. The integral in Eq. (42) depends on both the hadronic scale and the muon mass. The behavior of $\Pi^{\text {had }}\left(q^{2}\right)$ in the Euclidean domain is smooth and perturbative at large momenta. The region near the origin $q^{2}=0$ is a nonperturbative one. Thus, the basic objects that emerge in the analysis are derivatives of the function $\Pi^{\text {had }}(t)$ at the origin and integrals of the form

$$
\int_{m^{2}}^{\infty} \frac{d t}{t^{n}} \Pi^{\text {had }}(-t) \ln ^{p}\left(\frac{t}{m^{2}}\right)
$$

One can use this technique to avoid any reference to the physical region.

In model 1 given by Eqs. (25,27) the explicit interpolation function $p(t)$ is given in the whole complex $t$-plane. Therefore, it makes no difference how one computes the necessary integrals either in 
the Minkowskian or in the Euclidean domain. At the formal mathematical level the calculation with an explicit function can be performed in the spectral representation alone. The Euclidean approach will lead to the same formal results. For the calculation of the basic objects emerging in the spectral representation

$$
\mathcal{M}_{n, p}(\Delta)=\Delta^{n} \int_{\Delta}^{\infty} \frac{d s}{s^{n+1}} \ln ^{p}\left(\frac{s}{m^{2}}\right)
$$

the recurrence relation

$$
\mathcal{M}_{n, p}(\Delta)=\frac{1}{n} \ln ^{p}\left(\Delta / m^{2}\right)+\frac{p}{n} \mathcal{M}_{n, p-1}(\Delta)
$$

can be used to decrease a power of the logarithm in the integrand of Eq. (44). The results for the first two powers of the logarithm are

$$
\mathcal{M}_{n, 0}(\Delta)=\frac{1}{n}, \quad \mathcal{M}_{n, 1}(\Delta)=\frac{1}{n} \ln \left(\Delta / m^{2}\right)+\frac{1}{n^{2}} .
$$

Writing the analytical expression for the NLO vertex contribution to the MAMM in the form

$$
a_{\mu}^{\bmod }(\text { ver; NLO; analyt })=\left(\frac{\alpha}{\pi}\right)^{3} V\left(m^{2} / \Delta\right)
$$

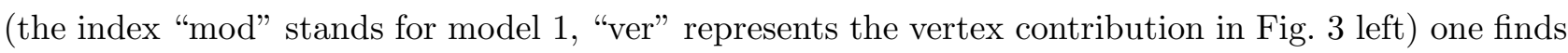

$$
V(v)=\frac{v}{9}\left(\frac{377}{18}-2 \pi^{2}+\frac{23}{6} \ln v\right)+\frac{v^{2}}{9}\left(\frac{23647}{1152}-\frac{37 \pi^{2}}{16}+\frac{677}{144} \ln v+\frac{19}{48} \ln ^{2} v\right)+o\left(v^{2}\right)
$$

where $o\left(v^{2}\right)$ is any function that satisfies $\lim _{v \rightarrow 0} o\left(v^{2}\right) / v^{2}=0$. For brevity we have explicitly presented only two terms of the expansion of the function $V\left(\mathrm{~m}^{2} / \Delta\right)$ at small $\mathrm{m}^{2} / \Delta$ resulting from the corresponding expansion of the kernel in Eq. (38). The result of evaluating the $u, d, s$ light mode contribution obtained from Eqs. (47,48), together with the appropriate QCD group factor $N_{c}\left(e_{u}^{2}+e_{d}^{2}+e_{s}^{2}\right)=2$ reads

$$
a_{\mu}^{\bmod }(\text { ver; NLO; light; analyt })=-191 \times 10^{-11} .
$$

The numerical integration of the kernel given in Eq. (38) results in the value

$$
a_{\mu}^{\bmod }(\text { ver; NLO; light })=-190(2) \times 10^{-11},
$$

to be compared with the corresponding results from the model of Ref. [35] which is based on the use of free massive fermions for computation of the interpolation function,

$$
a_{\mu}^{\bmod }(\text { ver; NLO; light })=-188 \times 10^{-11} .
$$

The total contribution of the vertex type including the heavy quarks reads

$$
a_{\mu}^{\bmod }(\operatorname{ver} ; \mathrm{NLO})=-194(3) \times 10^{-11},
$$

to be compared with the one obtained in the data-based approach 48

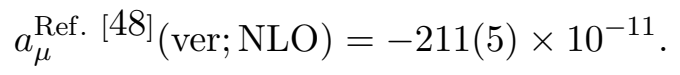

Note that the leading order contribution used in Ref. 48 is different from the value in Eq. (34). Therefore the direct comparison should be made with some caution. In fact, the value used for the 


\begin{tabular}{|r||c|c|c|}
\hline contribution & model 1 & model 2 & model 3 \\
\hline \hline$a_{\mu}^{\text {mod }}($ LO; light $)$ & $6908(114) \times 10^{-11}$ & $6908(114) \times 10^{-11}$ & $6578 \times 10^{-11}$ \\
\hline$a_{\mu}^{\text {mod }}($ LO; heavy $)$ & \multicolumn{3}{|c|}{$71(18) \times 10^{-11}$} \\
\hline \hline$a_{\mu}^{\text {mod }}($ ver; NLO; light $)$ & $-189.5(2.4) \times 10^{-11}$ & $-188.2(2.4) \times 10^{-11}$ & $-183.2 \times 10^{-11}$ \\
\hline$a_{\mu}^{\text {mod }}($ ver; NLO; heavy $)$ & \multicolumn{3}{|c|}{$-4.3(0.9) \times 10^{-11}$} \\
\hline$a_{\mu}^{\text {mod }}(\mathrm{db} ;$ NLO; light $)$ & $105.0(1.7) \times 10^{-11}$ & $104.9(1.7) \times 10^{-11}$ & $100.0 \times 10^{-11}$ \\
\hline$a_{\mu}^{\text {mod }}(\mathrm{db} ;$ NLO; heavy $)$ & \multicolumn{3}{|c|}{$10^{-11}$} \\
\hline \hline & $-87.7(0.7) \times 10^{-11}$ & $-86.5(0.7) \times 10^{-11}$ & $-86.4 \times 10^{-11}$ \\
\hline
\end{tabular}

Table 2: The different vacuum polarization type LO and NLO contributions for model 1 (second column), model 2 (third column), and model 3 (fourth column). The values for the effective masses for model 1 and model 2 are taken as $m_{\mathrm{eff}}=(201.0 \pm 1.9) \mathrm{MeV}$ and $m_{q}=(179.4 \pm 0.6) \mathrm{MeV}$ respectively, the cited uncertainties are a consequence of the uncertainties of these effective masses and of the masses of the heavy quarks. The error estimates due to these two sources can be added quadratically.

LO contribution in Ref. [48] is larger than the value in Eq. (34) which would result in a smaller value of $m_{\text {eff }}$ and consequently the larger value of the NLO vertex correction in the model calculation given in Eqs. (49,50). Nevertheless, the difference is within the error bars.

For a mixed contribution of the lepton-hadron type (so-called double bubble ("db") diagram) shown on the right hand side in Fig. 3 we find

$$
a_{\mu}^{\bmod }(\mathrm{db} ; \mathrm{NLO} ; \text { lept }+ \text { had })=106(2) \times 10^{-11}
$$

while the data-based estimate reads

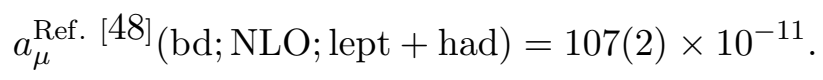

Thus, the results obtained with the interpolation model given in Eqs. (25,27) reproduce those obtained in the data-based approach.

The result for the total NLO hadronic contribution of the vacuum polarization type (two-point correlator only) is

$$
a_{\mu}^{\bmod }(\mathrm{vac} ; \mathrm{NLO})=-86.5(0.7) \times 10^{-11} .
$$

which has to be compared with the result shown in Eq. (37). All contributions, including the leading order contributions which reproduce the input of the determination of $m_{\text {eff }}$ are listed in the second column of Table 2 .

The model given in Eqs. (25,27) reproduces rather accurately the results for the NLO hadronic contributions found in the data-based analysis for the graphs related to vacuum polarization. This has been expected as these results are obtained by the integration of the two-point function with the NLO kernel $K^{(2)}(s)$ which has a structure very close to that of the leading order kernel $K(s)$. These two functions are shown in Fig. 田.

\section{Alternatives for the model spectral function}

The interpolation function for the two-point correlator of hadronic EM currents in Eq. (25) is very simple. One can use more sophisticated interpolations. A formal criterion for the choice of the inter- 


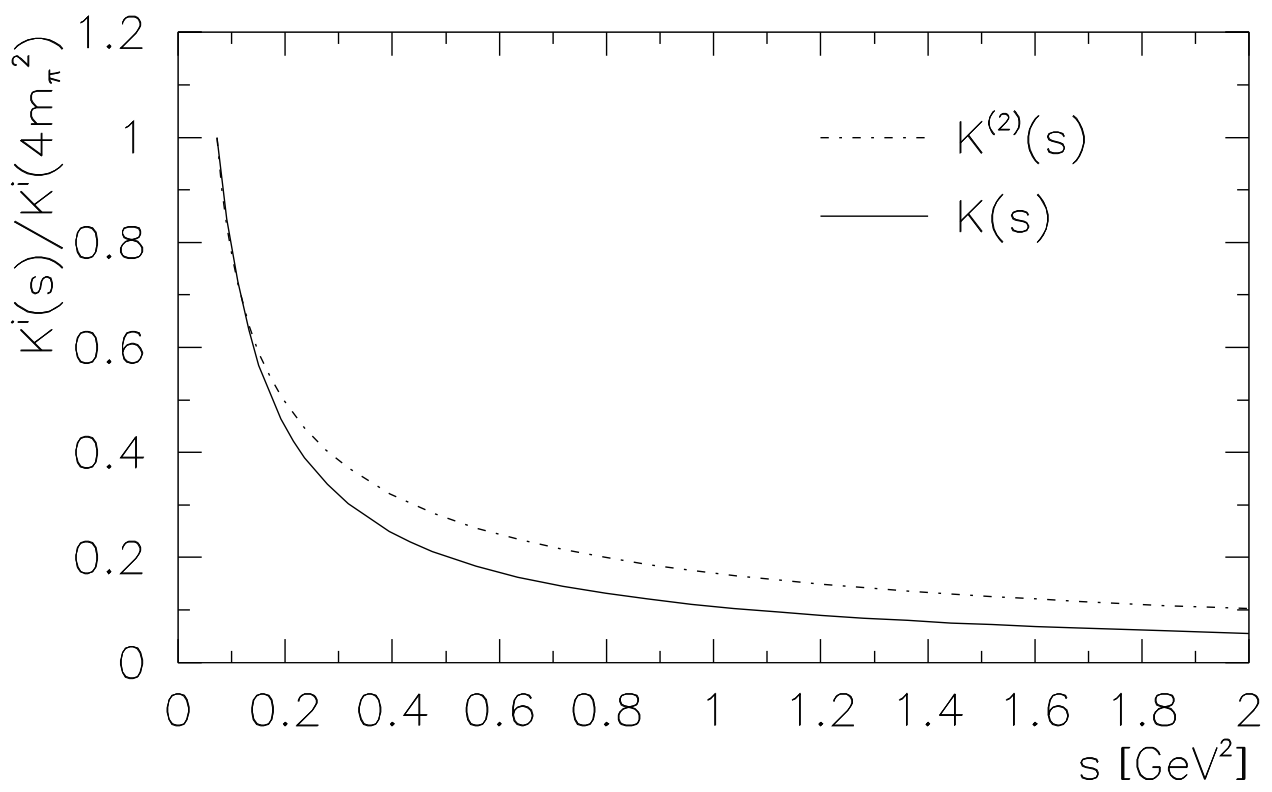

Figure 4: The LO and NLO kernels $K(s)$ and $K^{(2)}(s)$.

polation is its consistency with general principles of quantum field theory (analyticity and unitarity in this case). A practical criterion is its simplicity such that analytical calculations become technically feasible. One can turn to free field models in a search for mathematical functions that can be used in the interpolation procedure. For instance, the scalar or fermionic polarization functions with masses as free parameters can be taken as suitable candidates. The fermionic interpolation function was considered in detail in Ref. [35]. It is given by the expression

$$
\begin{gathered}
\pi\left(t, m_{q}\right)=\left(\frac{1}{3 z}-1\right) \varphi(z)-\frac{1}{9} \\
\varphi(z)=\frac{1}{\sqrt{z}} \operatorname{artanh}(\sqrt{z})-1, \quad z=\frac{t}{4 m_{q}^{2}+t} .
\end{gathered}
$$

The discontinuity across the cut $\left(4 m_{q}^{2}, \infty\right)$ at $t=-s-i 0$ is given by the fermionic spectral density of the form

$$
\rho_{q}(s)=\frac{1}{3} \sqrt{1-\frac{4 m_{q}^{2}}{s}}\left(1+\frac{2 m_{q}^{2}}{s}\right) .
$$

A pictorial representation of $\rho_{q}(s)$ is shown in Fig. 5. The two functions $f\left(t, m_{\mathrm{eff}}\right) / 3$ and $-d \pi\left(t, m_{q}\right) / d t$ coincide within $1 \%$ accuracy in the interval $t=\left(0, m_{q}^{2}\right)$ if the effective parameters are related through $m_{\text {eff }} / m_{q}=\sqrt{5} / 2 \approx 1.12$. This is expected from duality considerations based on the shape of the discontinuity across the cut given in Eqs. (28,58). Since the integral in Eq. (29) is saturated at the scale of the order of the muon mass $\sqrt{t} \sim m_{\mu}$ and $m_{\mu}<m_{\text {eff }}$, the closeness of the integrated interpolation functions implies a rather accurate equality of the resulting integrals. The generalization to interpolations based on scalar field theories is straightforward. The interpolation given by the spectral density in Eq. (58) will be referred to as model 2 in the following.

At this stage it seems that hadrons have completely disappeared from the analysis and some artificial functions are rather arbitrarily used to compute the relevant integrals. The link to physics 
is that for such kind of inclusive observables as the MAMM which are sensitive to a contribution of many hadrons the analysis can completely be done in the Euclidean domain with the only IR sensitive contribution coming from the region near the origin. In the Euclidean domain the contribution of all hadrons to the MAMM is smeared to the extent which is determined by the distance between the integration region and the nearest physical singularity. The hadronic singularity is taken from experiment as a two-pion cut with the kinematical constraint $s>4 m_{\pi}^{2}$. The integration, in fact, is sensitive to scales $\sqrt{t} \sim 2 m_{\mu}$. Therefore, a correctly normalized function (as for duality at large energies) with the appropriate IR behavior in the interval $0<t<4 m_{\mu}^{2}$ reproduces the data with reasonable accuracy. The IR behavior is mainly determined by the experimental scale $4 m_{\pi}^{2}$.

To illustrate this statement let us consider a more realistic interpolation for the vacuum polarization function in the Euclidean domain from the point of view of experimental hadron physics than those described so far. The model hadron spectrum for light modes can be chosen in the following simple form

$$
\rho^{\text {had }}(s)=2 m_{\rho}^{2} \delta\left(s-m_{\rho}^{2}\right)+\theta\left(s-2 m_{\rho}^{2}\right) .
$$

This is a one-scale no-parameter model that satisfies the duality constraints from the operator product expansion [7, 8, 49]. The hadronic scale of the model is given by the $\rho$-meson mass $m_{\rho}$ which is eventually fixed from experiment [16]. We neglect small violations of flavor symmetry for the $u, d, s$ light modes and consider them to be degenerate. The spectrum from Eq. (59) gives an interpolation function of the form

$$
f^{\text {had }}(t)=\frac{2 m_{\rho}^{2}}{\left(t+m_{\rho}^{2}\right)^{2}}+\frac{1}{t+2 m_{\rho}^{2}} .
$$

The value of the interpolation function $f^{\text {had }}(t)$ at the origin $t=0$ reads

$$
f^{\text {had }}(0)=\frac{5}{2 m_{\rho}^{2}}
$$

It should be compared with the value from Eq. (30). For $m_{\rho}=769.9 \mathrm{MeV}$ one finds $f^{\text {had }}(0)=$ $4.22 \mathrm{GeV}^{-2}$ while the data give $f(0)=1 / \Delta=6.25 \mathrm{GeV}^{-2}$. This is fairly reasonable given the simplicity of the model but is not accurate enough. Note that there is no single free parameter in the model given in Eq. (59) and the shape of the spectrum is fixed from the duality constraint at large energies. The weight function $F(t)$ from Eqs. (20,21) determining the MAMM integral can resolve the behavior of the interpolation function $f^{\text {had }}(t)$ for the hadron correlator at the scales of order $m_{\mu}=105.66 \mathrm{MeV}$. However, the approximation of infinitely narrow resonance in Eq. (59) is too rough for computing such an integral. Thus, the low energy behavior of the spectrum is not precise enough which leads to an insufficient accuracy of the interpolation function at small $t$. Formally this is seen in the absence of the scale $4 m_{\pi}^{2}$ which is known to be important for the evaluation of the integral in the data-based analysis. Therefore the spectrum from Eq. (59) should be corrected for this particular application. A dominant role in the data-based analysis is played by two-pion states. The $\rho$ meson is a resonance in the two-pion system therefore its contribution effectively takes into account the pion singularity as well. However, the zero width approximation is not good enough for computing the particular integral in Eq. (20). A natural modification of the spectrum is to introduce a finite width for the $\rho$ meson. This is achieved by replacing the function $\delta\left(s-m_{\rho}^{2}\right)$ by the Breit-Wigner function for the resonance part of the spectrum in Eq. (59)

$$
\rho_{\mathrm{R}}^{\mathrm{had}}(s)=\frac{2 m_{\rho}^{2}}{\pi} \frac{\Gamma_{\rho} m_{\rho}}{\left(s-m_{\rho}^{2}+\Gamma_{\rho}^{2} / 4\right)^{2}+\Gamma_{\rho}^{2} m_{\rho}^{2}},
$$



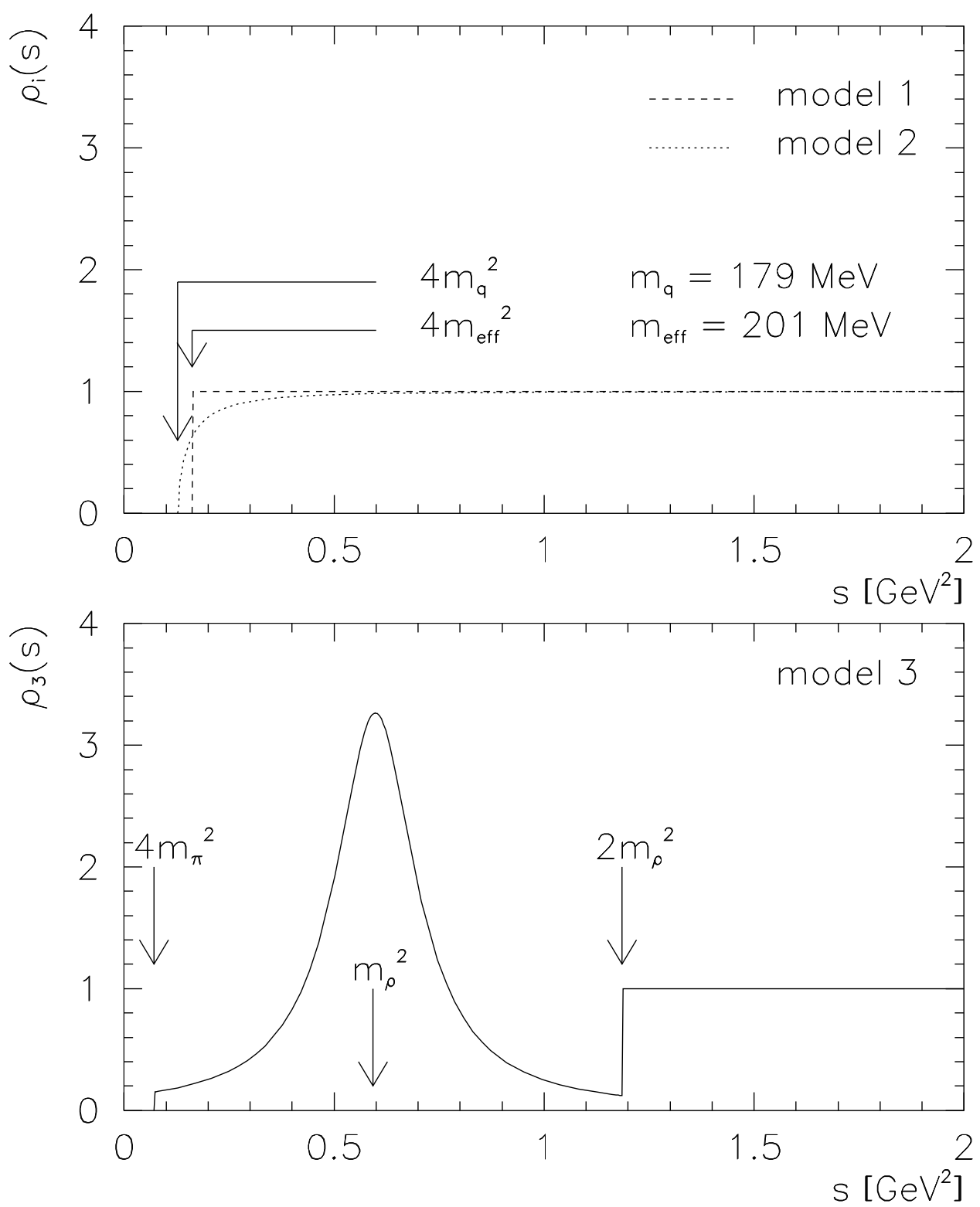

Figure 5: $s$-dependence of the spectral functions $\rho_{1}(s)=r(s)$ of model 1 in Eq. (28) and $\rho_{2}(s)=3 \rho_{q}(s)$ of model 2 in Eq. (58) (upper diagram), as compared to the spectral function $\rho_{3}(s)=\rho^{\text {had }}(s)$ for model 3 in Eqs. (59,62). We use $m_{\mathrm{eff}}=201 \mathrm{MeV}, m_{q}=179 \mathrm{MeV}$, and the central values $m_{\rho}=$ $769.9 \mathrm{MeV}$ and $\Gamma_{\rho}=150.2 \mathrm{MeV}$ [16]. 


$$
\rho_{\Gamma}^{\mathrm{had}}(s)=\theta\left(s-4 m_{\pi}^{2}\right) \theta\left(2 m_{\rho}^{2}-s\right) \rho_{\mathrm{R}}^{\mathrm{had}}(s)+\theta\left(s-2 m_{\rho}^{2}\right) .
$$

The interpolation function based on this spectrum will be called model 3 in the following. Fig. 5 shows the $s$-dependence of $\rho_{\Gamma}^{\text {had }}(s)$. The expression for the resonance part of the spectrum reduces to $2 m_{\rho}^{2} \delta\left(s-m_{\rho}^{2}\right)$ in the limit $\Gamma_{\rho} \rightarrow 0$. Using the Breit-Wigner form of the spectrum for the region $4 m_{\pi}^{2}<s<2 m_{\rho}^{2}$ one finds the contribution of the resonance to the interpolation function in the Euclidean domain

$$
f_{\mathrm{R}}^{\mathrm{had}}(t)=\int_{4 m_{\pi}^{2}}^{2 m_{\rho}^{2}} \frac{\rho_{\mathrm{R}}^{\mathrm{had}}(s) d s}{(s+t)^{2}} .
$$

The interpolation function in the Euclidean domain for the spectrum with nonzero width reads

$$
f_{\Gamma}^{\mathrm{had}}(t)=f_{\mathrm{R}}^{\mathrm{had}}(t)+\frac{1}{t+2 m_{\rho}^{2}}
$$

Computing the value of the interpolation function at the origin for $\Gamma_{\rho}=150.2 \mathrm{MeV}$ [16] one finds

$$
f_{\Gamma}^{\mathrm{had}}(0)=f_{\mathrm{R}}^{\mathrm{had}}(0)+\frac{1}{2 m_{\rho}^{2}}=5.15+0.84=6.0 \mathrm{GeV}^{-2}
$$

instead of the result (61) obtained in the infinitely narrow resonance approximation. The number from Eq. (65) differs from the data-based estimate $f(0)=1 / \Delta=6.25 \mathrm{GeV}^{-2}$ by $4 \%$ only. Note that no free parameters have been used so far for the description of the hadron spectrum. The integrals entering the MAMM as in Eq. (29) are also rather close. One finds

$$
I^{\text {data }}=0.0194
$$

to be compared with

$$
I_{\Gamma}^{\mathrm{had}}=0.0155+0.0030=0.0185
$$

where the first term in the sum is given by the resonance and the second by the continuum contribution. Thus, the simple and parameter-free model from Eqs. (62,63) already gives a reasonable precision of about $5 \%$ for the LO hadronic contribution to the MAMM. In this sense it successfully incorporates experimental information necessary for the MAMM computation.

The spectral functions taken for model 1 from Eq. (28), for model 2 from Eq. (58), and for model 3 from Eq. (62) are shown in Fig. 5 in order to allow one to compare these models. Neither model 1 nor model 2 has a discontinuity across the positive semiaxis of the $s$-plane resembling the experimental spectrum. However, both models result in integrals over the spectrum for the respective kernels which are very close to the result obtained in the experimentally inspired model 3 and, eventually, to the data represented in Eq. (66). From the purely mathematical point of view this is related to the fact that the procedure of analytic continuation is an incorrectly posed problem: small variations of functions in the Euclidean domain can produce big variations on the cut. The $t$-dependence of the Euclidean representation by the functions $f(t),-3 d \pi(t) / d t$, and $f_{\Gamma}^{\text {had }}(t)$ is shown in Fig. 6. A phenomenological interpretation of the situation consists in a duality between hadrons and free light fermions with QCD quantum numbers as for the particular application related to the MAMM computation. Of course, the main objective of the calculation of the hadronic contribution at the leading order from experiment is to reach a high precision. The use of direct data seems to be superior to a parameterization of 

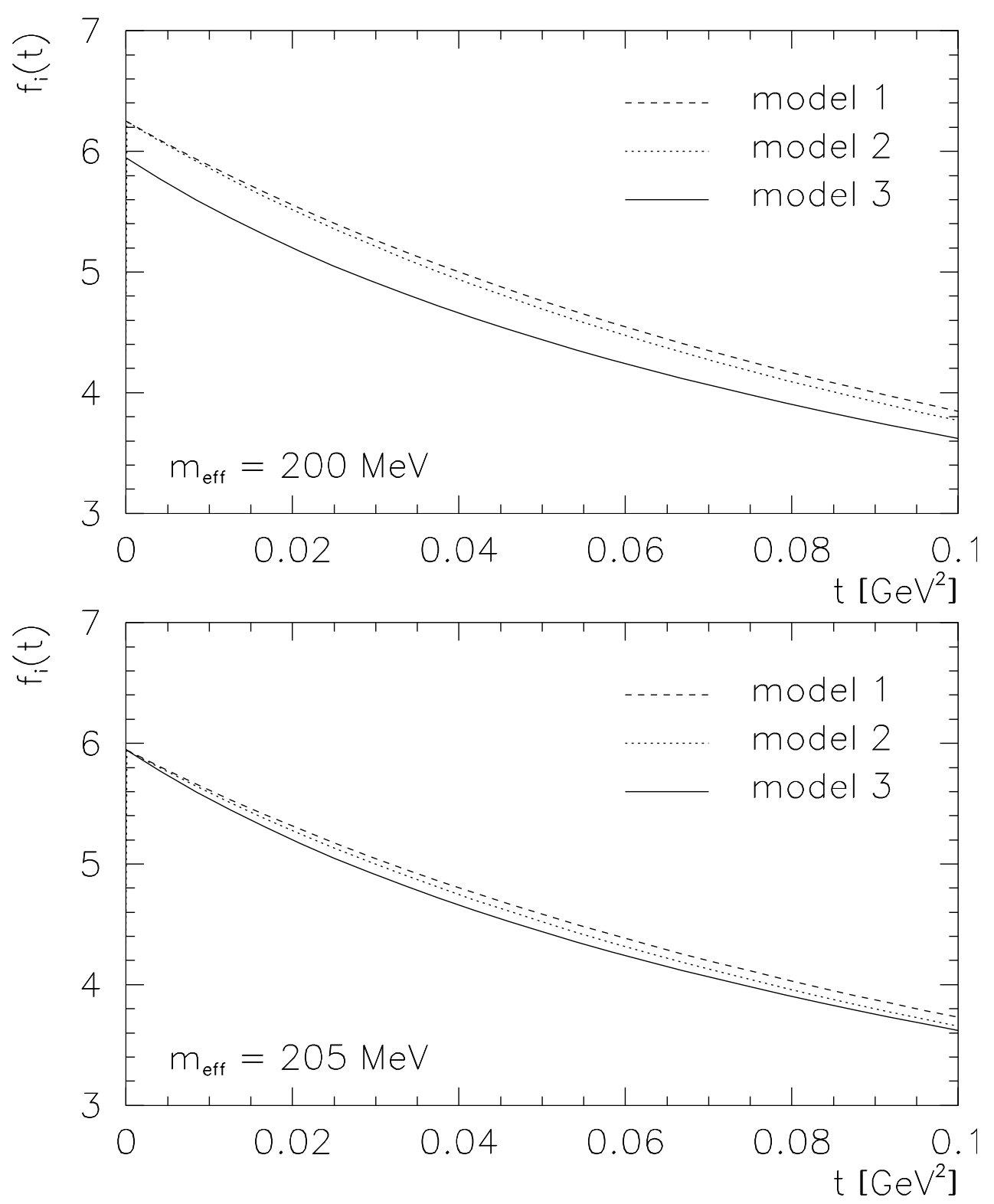

Figure 6: The functions $f_{i}(t)$ for the three different models where $f_{1}(t)=f(t)$ is given by Eq. (25), $f_{2}(t)=-3 d \pi(t) / d t$ is given by Eq. (57) and $f_{3}(t)=f_{\Gamma}^{\text {had }}(t)$ is given by Eq. (64). We take $\Delta=4 m_{\text {eff }}^{2}$ where $m_{\text {eff }}=200 \mathrm{MeV}$ is used for the upper diagram and $m_{\mathrm{eff}}=205 \mathrm{MeV}$ for the lower diagram. The parameter $m_{q}$ used for $\pi(t)$ is connected to $m_{\mathrm{eff}}$ by $m_{q}=2 m_{\mathrm{eff}} / \sqrt{5}$. The values $m_{\rho}=769.9 \mathrm{MeV}$ and $\Gamma_{\rho}=150.2 \mathrm{MeV}$ used in $f_{R}^{\text {had }}$ are taken from Ref. [16]. 

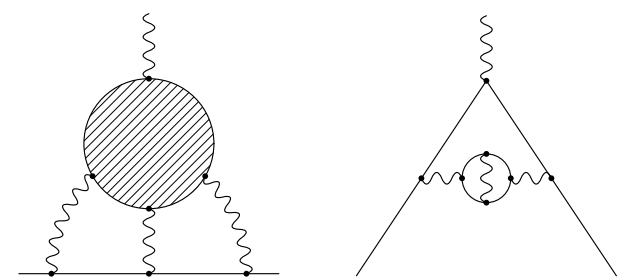

Figure 7: The light-by-light contribution (left) and the two-photon Green function (right) to the MAMM at NLO

the spectrum from indirect observations. However, as soon as the integral over the data is computed a smooth interpolation function of a simple form can be introduced in the Euclidean domain to be used in higher order calculations. Because this interpolation function is explicit and complies with the general properties of analyticity and unitarity one can find its discontinuity across the positive semiaxis and perform further calculations in the spectral representation as well. The analysis of NLO contributions along these lines shows that the data-based results are accurately reproduced [35].

\section{Discussion of the light-by-light contribution}

Still the class of interpolation models based on field theories contains more than just a useful set of functions with suitable properties. It can be used, with some caution, for extrapolations to higher order correlation functions as well. Indeed, the interpolation models Eqs. (27,57) suffice for the calculation of the polarization-type hadronic contributions to the MAMM related to the two-point correlator of hadronic EM currents. For the whole computation at NLO one needs a four-point correlator of hadronic currents. It appears in two instances: as a contribution to the light-by-light graph and a two-photon Green function (see Fig. 7). For the contributions to the two-photon Green function the relevant projection of the four-point correlator depends on one external momentum and the above discussion is applicable theoretically as the interpolation function of one complex variable is necessary. There is no experimental data to fix the IR scale though. For the light-by-light graph the projection of the four-point correlator relevant for the MAMM calculation is a function of two independent fourmomenta $k_{1}, k_{2}$. The scalar form factors $g_{4}\left(k_{1}^{2}, k_{2}^{2},\left(k_{1}-k_{2}\right)^{2}\right)$ of the projection of the tensor four-point correlator relevant for the MAMM computation are given by

$$
\begin{array}{r}
i^{2} \int\left\langle T x^{\omega} j_{\mu}^{h a d}(x) j_{\nu}^{h a d}(y) j_{\tau}^{h a d}(z) j_{\sigma}^{h a d}(0)\right\rangle e^{i k_{1} y+i k_{2} z} d x d y d z \\
=\sum_{i} \mathcal{T}_{\mu \nu \tau \sigma}^{\omega i}\left(k_{1}, k_{2}\right) g_{4}^{i}\left(k_{1}^{2}, k_{2}^{2},\left(k_{1}-k_{2}\right)^{2}\right) .
\end{array}
$$

The functions $g_{4}^{i}$ are functions of the three scalar variables $k_{1}^{2}, k_{2}^{2}$, and $\left(k_{1}-k_{2}\right)^{2}$. Here $\mathcal{T}_{\mu \nu \tau \sigma}^{\omega i}\left(k_{1}, k_{2}\right)$ are tensor structures which are polynomials in the four-momenta $k_{1}, k_{2}$ and the metric tensor $g_{\mu \nu}$. The form factors $g_{4}^{i}$ depend on the IR hadronic scale $M_{h}$ which is related to the experimental masses of light hadrons (pions). It may effectively differ from the scale emerging in the two-point correlator. These IR scales cannot be found theoretically in QCD as the regime of strong coupling is not treatable in perturbation theory. They can perhaps be estimated in some nonperturbative approach as, for example, the lattice approximation. The scalar form factors $g_{4}^{i}\left(k_{1}^{2}, k_{2}^{2},\left(k_{1}-k_{2}\right)^{2} ; M_{h}\right)$ are integrated over $k_{1}, k_{2}$ with the weight functions $w\left(k_{1}, k_{2}, m\right)$ to give a contribution to the MAMM in a full analogy 
with the two-point correlator

$$
\sum_{i} \int g_{4}^{i}\left(k_{1}^{2}, k_{2}^{2},\left(k_{1}-k_{2}\right)^{2} ; M_{h}\right) w^{i}\left(k_{1}, k_{2}, m\right) d^{4} k_{1} d^{4} k_{2} .
$$

The weight functions $w^{i}\left(k_{1}, k_{2}, m\right)$ are generated by perturbative diagrams and depend on integration momenta $k_{1}, k_{2}$ in the loops and on the muon mass $m$ (more precisely, they depend also on the muon momentum $p$ which is taken on the muon mass-shell $p^{2}=m^{2}$ ). To the leading order of expansion in $m / M_{h}$ the integration in explicit models reduces to vacuum three loop bubbles which can be done analytically [50].

The calculation of the integral in Eq. (69) within an hadronization procedure requires one to establish the analytic properties of a given form factor $g_{4}^{i}\left(z_{1}, z_{2}, z_{3} ; M_{h}\right)$ as a function of three complex variables $z_{i}$. Also one has to saturate the singularities with the contributions of hadrons in full analogy with the two-point correlator. Then the integral in Eq. (69) is calculated through the discontinuities on the singularities. In other words, one can use the dispersion representation for the form factors $g_{4}^{i}\left(k_{1}^{2}, k_{2}^{2},\left(k_{1}-k_{2}\right)^{2} ; M_{h}\right)$ with some spectral density and then integrate over the momenta $k_{1}, k_{2}$ explicitly. At this point one is left with an integral over the whole physical spectrum for the form factors with kernels obtained after such integration. Note that this is close to the way how the actual integration of the light-by-light diagram was done analytically for leptons where the physical spectrum can be computed in perturbation theory [19].

While the physical spectrum at low energies cannot be computed in QCD perturbation theory point-wise, the integrals over the hadronic spectrum for the three-point functions are quite accurately calculated in the sum rule approach based on duality between hadron and quark-gluon contributions [51]. As one needs only integrals of the functions $g_{4}^{i}\left(k_{1}^{2}, k_{2}^{2},\left(k_{1}-k_{2}\right)^{2} ; M_{h}\right)$ one can avoid constructing these functions point-wise. Assuming general requirements of smoothness one can use an interpolation function in the Euclidean domain. Recently it has been argued that the neutral pion contribution to the amplitude Eq. (69) does not give a singular dominant contribution by itself in the kinematical region relevant for the MAMM calculation and can be treated within the duality approach [35] (see also Ref. [52]). This implies that the integration over the Euclidean domain properly takes into account singularities of the $g_{4}^{i}$-functions related both to the pion contribution and twoparticle cuts given by the two-pion states. Thereby all contributions at low energies of the integrand (spectrum) are taken into account, for example, the $\rho$ meson. The integral of the spectrum can therefore be approximated by an integral of some interpolation function with an appropriate IR parameter. As the IR scale is set by the pion mass $m_{\pi}$ one would expect a numerical value of the IR parameter to be of the same order of magnitude. In contrast to the two-point correlator $\Pi^{\text {had }}\left(q^{2}\right)$ the $g_{4}^{i}$-functions related to the four-point correlator in Eq. (68) are complicated functions of three complex variables which makes it difficult to find an appropriate candidate for an interpolation function obeying all general requirements of field theory. A simple way to find such an appropriate interpolation is to use a field theory for constructing the spectrum. The use of a free fermionic theory is just the way one can generate the interpolation function having the necessary properties. Indeed, it does not violate any general principles (gauge invariance, discrete symmetries, analyticity, unitarity, ... ) as the finite order perturbative field theory is about the only realistic field theory that is known to obey the general principles. One can consider such an interpolation as an efficient adaptive integration procedure like the spline technique or Monte Carlo routine VEGAS [53]. It is not a full-scale approximation of QCD at low energies that would allow one to compute the exclusive characteristics of hadrons, i.e. to com- 
pute the functions $g_{4}^{i}\left(k_{1}^{2}, k_{2}^{2},\left(k_{1}-k_{2}\right)^{2} ; M_{h}\right)$ point-wise. It is a way to find integrals of the special type in Eq. (69) using duality between quarks and hadrons. Note that the duality idea between $s$-channel resonances and Regge trajectories was very useful in description of hadron scattering before the invention of QCD. Thus, many model interpolations are possible in the leading order and give rather accurate results. However, in the higher orders of EM perturbation theory there are strict constraints on the interpolation functions that one can use. Indeed, it is difficult to generalize the models given in Eqs. (25,63) to higher orders of perturbation theory. The first model is rather artificial indeed and is not literally realized in any field theory while the second requires to work out the full-scale hadronization of QCD for the four-point correlator already at NLO. This is technically difficult as the data-based approach shows [33, 54]. At the same time the model given in Eq. (57) for the two-point correlator can immediately be extended to any order of perturbation theory in the electromagnetic interaction as it is a free fermion theory with $m_{q}$ being an IR regulator. One also knows that this model is accurate for the LO hadronic contributions with the IR scale taken from the data. The IR parameter effectively estimates the distance from the Euclidean domain of integration to the physical singularities of the hadronic correlation functions. The key physical point of the quantitative analysis of the hadronic contributions to the MAMM within this approach is that the same parameter $m_{\text {eff }}$, or $m_{q}$, enters both two-point and four-point correlators. This is, of course, an assumption which is based on the observation that the numerical value of this parameter is close to the pion mass. The absence of the neutral pion pole in the $g_{4}^{i}$ functions is essential for such an assumption to be valid. The result of the analysis of the total next-to-leading order hadronic contributions to the MAMM based on the fermionic interpolation with the same value of the effective IR scale $M_{h}=m_{q}$ for the two-point (polarization type) and four-point (light-by-light) correlators [35]

$$
a_{\mu}^{\bmod }(\mathrm{NLO})=(85 \pm 20) \times 10^{-11}
$$

agrees with the experimental value from Eq. (15). Note that this result includes the explicit contribution of the EM correction to the polarization function at the NLO, i.e. the basic normalization quantity obtained from the data as given in Eq. (34) is supposed not to contain this type of contributions. This can be a rather clumsy arrangement from the experimental point of view but it is more definite concerning the theoretical quantities. Numerically the difference is well within error bars for the LO contribution though.

If the scalar field theory of charged pions given by the Lagrangian

$$
L_{\pi}=\left|D_{\mu} \pi\right|^{2}-m_{\pi}^{2} \pi^{2}, \quad D_{\mu}=\partial_{\mu}-i e A_{\mu}
$$

is used to generate the four-point correlator of hadronic currents and thereby the form factors $g_{4}^{i}$ that enter as integrands in Eq. (69) then the IR scale is explicitly identified with the pion mass and can be taken from experiment. In this case one has to add the contribution of higher resonances to satisfy the duality constraints at large energies which would make this approach equivalent to the one based on explicit hadronization. The free fermionic theory with the QCD arrangement of quantum numbers is exceptional in this sense as it automatically complies with the duality constraints at large energies. One, of course, should remember that this is a model and its large energy behavior is accurate only up to higher order QCD corrections. For the applications of interest this is inessential. A technical advantage of the fermionic theory is that the analytical results for the MAMM at NLO are available. For the scalar theory numerical results are available at present although the calculations can 
in principle be done analytically as well since all necessary master integrals have been found [19, 55]. They have already been used in three loop calculations. According to the hadronization picture the contributions of the fermionic interpolation functions at low energies should be substituted by the pionic ones. In the pure fermionic model with a small effective mass the replacement of the hadronic contributions by the model ones is effectively done at rather low energies which makes the separate contribution of pions small or even vanishing.

\section{Summary and conclusions}

A parameterization of the photon vacuum polarization function related to the light hadronic modes is described in the Euclidean domain. The model contains a single parameter which is fixed from the experimental result for the LO hadronic contribution to the MAMM. The model describes the NLO hadronic contributions of the vacuum polarization-type in agreement with existing estimates. The calculation of the total NLO hadronic contribution to the MAMM in a closely related model based on the fermionic interpolation of correlators of hadronic electromagnetic currents is also discussed.

\section{Acknowledgments}

We thank K. Chetyrkin for his interest in the work and useful discussions. This work is partially supported by the Russian Fund for Basic Research under contract 99-01-00091 and 01-02-16171 and by the INTAS grant. A.A.P. as well as S.G. gratefully acknowledge grants given by the Deutsche Forschungsgemeinschaft.

\section{Note added}

Recently the authors of Ref. [22] have updated their result concerning the neutral pion contribution to the light-by-light diagram. While the magnitude of the numerical value remains the same, the sign of the contribution has been changed [56]. This change brings the explicit hadron-based result for the light-by-light contribution rather close to that obtained within the duality approach of Ref. [35] which has further been investigated and developed in the present paper (originally posted at the hep-ph ArXiv as hep-ph/0111206). Thus, the present theoretical result for the muon anomalous magnetic moment agrees with the current experimental value.

\section{References}

[1] D. J. Gross and F. Wilczek, Phys. Rev. Lett. 30, 1343 (1973);

H. D. Politzer, Phys. Rev. Lett. 30, 1346 (1973).

[2] S. Weinberg, Phys. Rev. Lett. 18, 188 (1967).

[3] S. Weinberg, Physica 96A, 327 (1979).

[4] J. Gasser and H. Leutwyler, Annals Phys. 158, 142 (1984), Nucl. Phys. B250, 465 (1985).

[5] G. F. Chew, The analytic S-matrix, 1966. 
[6] S. L. Adler, Phys. Rev. D10, 3714 (1974); A. De Rujula and H. Georgi, Phys. Rev. D13, 1296 (1976); E. C. Poggio, H. R. Quinn and S. Weinberg, Phys. Rev. D13, 1958 (1976); R. Shankar, Phys. Rev. D15, 755 (1977).

[7] M. A. Shifman, A. I. Vainshtein and V. I. Zakharov, Nucl. Phys. B147, 385 (1979); V. A. Novikov, M. A. Shifman, A. I. Vainshtein and V. I. Zakharov, Nucl. Phys. B191, 301 (1981).

[8] N. V. Krasnikov, A. A. Pivovarov and A. N. Tavkhelidze, JETP Lett. 36, 333 (1982), Z. Phys. C19, 301 (1983).

[9] L. J. Reinders, H. Rubinstein and S. Yazaki, Phys. Rept. 127, 1 (1985).

[10] E. Braaten, Phys. Rev. Lett. 60, 1606 (1988); S. Narison and A. Pich, Phys. Lett. B211, 183 (1988); E. Braaten, S. Narison and A. Pich, Nucl. Phys. B373, 581 (1992).

[11] A. A. Pivovarov, Sov. J. Nucl. Phys. 54, 676 (1991), Z. Phys. C53, 461 (1992); F. Le Diberder and A. Pich, Phys. Lett. B 289, 165 (1992); S. Groote, J. G. Körner and A. A. Pivovarov, Phys. Lett. B407, 66 (1997), Mod. Phys. Lett. A13, 637 (1998).

[12] P. Colangelo and A. Khodjamirian, arXiv:hep-ph/0010175.

[13] A. J. Buras, "Flavour physics and CP violation in the SM," arXiv:hep-ph/0109197; S. Bosch, A. J. Buras, M. Gorbahn, S. Jager, M. Jamin, M. E. Lautenbacher and L. Silvestrini, Nucl. Phys. B565, 3 (2000); M. Ciuchini et al., JHEP 0107, 013 (2001).

[14] A. A. Penin and A. A. Pivovarov, Phys. Rev. D49, 265 (1994), Int. J. Mod. Phys. A10, 4065 (1995); J. F. Donoghue, Nucl. Phys. Proc. Suppl. 96, 329 (2001); J. O. Eeg, "Theoretical estimate(s) of the CP-violating quantity epsilon'/epsilon in K $\rightarrow$ 2pi decays," arXiv:hep-ph/0010042.

[15] A. Pich and E. De Rafael, Phys. Lett. B158, 477 (1985); K. G. Chetyrkin, A. L. Kataev, A. B. Krasulin and A. A. Pivovarov, Phys. Lett. B174, 104 (1986); L. J. Reinders and S. Yazaki, Nucl. Phys. B288, 789 (1987); J. Prades, C. A. Dominguez, J. A. Penarrocha, A. Pich and E. de Rafael, Z. Phys. C51, 287 (1991); J. Bijnens and J. Prades, Nucl. Phys. B444, 523 (1995); A. A. Ovchinnikov and A. A. Pivovarov, Sov. J. Nucl. Phys. 48, 120 (1988), Phys. Lett. B207, 333 (1988); A. Pich, Phys. Lett. B206, 322 (1988); S. Narison and A. A. Pivovarov, Phys. Lett. B327, 341 (1994).

[16] C. Caso et al., [Particle Data Group Collaboration], Eur. Phys. J. C3, 1 (1998); D. E. Groom et al. [Particle Data Group Collaboration], Eur. Phys. J. C15, 1 (2000).

[17] H. N. Brown et al., hep-ex/0009029, hep-ex/0102017.

[18] A. Czarnecki and W. J. Marciano, Phys. Rev. D64, 013014 (2001).

[19] S. Laporta and E. Remiddi, Phys. Lett. B301, 440 (1993).

[20] T. Kinoshita, B. Nizic, Y. Okamoto, Phys. Rev. D41, 593 (1990).

[21] P. J. Mohr and B. N. Taylor, Rev. Mod. Phys. 72, 351 (2000). 
[22] M. Hayakawa and T. Kinoshita, Phys. Rev. D57, 465 (1998).

[23] T. Kinoshita, Rept. Prog. Phys. 59, 1459 (1996).

[24] S. J. Brodsky and J. D. Sullivan, Phys. Rev. 156, 1644 (1967); R. Jackiw and S. Weinberg, Phys. Rev. D5, 2473 (1972); T. V. Kukhto, E. A. Kuraev, Z. K. Silagadze and A. Schiller, Nucl. Phys. B371, 567 (1992); A. Czarnecki, B. Krause and W. J. Marciano, Phys. Rev. D52, 2619 (1995); S. Peris, M. Perrottet and E. de Rafael, Phys. Lett. B355, 523 (1995).

[25] J. Gasser and H. Leutwyler, Phys. Rept. 87, 77 (1982); S. Narison, Nucl. Phys. Proc. Suppl. 86, $242(2000)$.

[26] R. Gupta and K. Maltman, "Light quark masses: A status report at DPF 2000," arXiv:hepph/0101132; K. Maltman, R. Gupta and T. Bhattacharya, Nucl. Phys. A631, 497C (1998); T. Bhattacharya, R. Gupta and K. Maltman, Phys. Rev. D57, 5455 (1998).

[27] S. Narison and E. de Rafael, Phys. Lett. B103, 57 (1981); C. Becchi, S. Narison, E. de Rafael and F. J. Yndurain, Z. Phys. C8, 335 (1981); A. L. Kataev, N. V. Krasnikov and A. A. Pivovarov, Phys. Lett. B123, 93 (1983), Nuovo Cim. A76, 723 (1983); A. Pich and J. Prades, JHEP 9806, 013 (1998); K. G. Chetyrkin, J. H. Kühn and A. A. Pivovarov, Nucl. Phys. B533, 473 (1998); J. G. Körner, F. Krajewski and A. A. Pivovarov, Eur. Phys. J. C20, 259 (2001).

[28] T. Kinoshita, B. Nizic, Y. Okamoto, Phys. Rev. D31, 2108 (1985).

[29] F. Jegerlehner, "Hadronic contributions to the photon vacuum polarization and their role in precision physics," arXiv:hep-ph/0104304.

[30] C. F. Cho, J. J. Sakurai, Phys. Lett. B30, 119 (1969); J. J. Sakurai, Phys. Lett. B46, 207 (1973).

[31] A. A. Pivovarov, Sov. J. Nucl. Phys. 54, 878 (1991), Int. J. Mod. Phys. A7, 6523 (1992).

[32] M. Hayakawa, T. Kinoshita and A. I. Sanda, Phys. Rev. Lett. 75, 790 (1995), Phys. Rev. D54, 3137 (1996).

[33] J. Bijnens, E. Pallante and J. Prades, Phys. Rev. Lett. 75, 1447 (1995) [Erratum-ibid. 75, 3781 (1995)], Nucl. Phys. B474, 379 (1996).

[34] J. G. Körner, A. A. Pivovarov and K. Schilcher, Eur. Phys. J. C9, 551 (1999); A. A. Pivovarov, "Running electromagnetic coupling constant: low-energy normalization and the value at $\mathrm{M}(\mathrm{Z})$." MZ-TH-00-51, Nov 2000. 53pp. hep-ph/0011135

[35] A. A. Pivovarov, arXiv:hep-ph/0110248.

[36] S. Eidelman and F. Jegerlehner, Z. Phys. C67, 585 (1995).

[37] S. Brodsky, E. de Rafael, Phys. Rev. 168, 1620 (1968);

M. Gourdin, E. de Rafael, Nucl. Phys. B10, 667 (1969).

[38] R. Alemany, M. Davier, A. Höcker, Eur. Phys. J. C2, 123 (1998)

[39] M. Davier, A. Höcker, Phys. Lett. B435, 427 (1998). 
[40] H. Lehmann, Nuovo Cim. 11, 342 (1954).

[41] N. V. Krasnikov, Rept. Math. Phys. 17, 309 (1980).

[42] A. A. Pivovarov, "Spectrality, coupling constant analyticity and the renormalization group," MZTH-01-13, Apr 2001. 19pp. [hep-ph/0104213]; S. Groote, J. G. Körner and A. A. Pivovarov, Phys. Rev. D 65, 036001 (2002) hep-ph/0105227.

[43] B. E. Lautrup, A. Peterman, E. de Rafael, Phys. Rep. 3, 193 (1972).

[44] M. A. Samuel and G. Li, Phys. Rev. D44, 3935 (1991).

[45] J. H. Kühn and M. Steinhauser, Nucl. Phys. B 619, 588 (2001); S. Narison, Phys. Lett. B520, 115 (2001).

[46] J. H. Kühn, A. A. Penin and A. A. Pivovarov, Nucl. Phys. B534, 356 (1998); A. A. Penin and A. A. Pivovarov, Phys. Lett. B435, 413 (1998); Nucl. Phys. B549, 217 (1999).

[47] R. Barbieri and E. Remiddi, Nucl. Phys. B90, 233 (1975).

[48] B. Krause, Phys. Lett. B390, 392 (1997).

[49] N. V. Krasnikov and A. A. Pivovarov, Phys. Lett. B112, 397 (1982); A.A. Pivovarov, Phys. Atom. Nucl. 62, 1924 (1999).

[50] D. J. Broadhurst, Eur. Phys. J. C8, 311 (1999).

[51] V. A. Nesterenko and A. V. Radyushkin, Phys. Lett. B115, 410 (1982); Phys. Lett. B128, 439 (1983); JETP Lett. 39, 707 (1984); A. A. Pivovarov, JETP Lett. 55, 6 (1992), Int. J. Mod. Phys. A10, 3125 (1995).

[52] A. A. Pivovarov, Phys. Lett. B255, 117 (1991), Nucl. Phys. B396, 119 (1993), Phys. Rev. D47, $5183(1993)$.

[53] G. P. Lepage, "Vegas: An Adaptive Multidimensional Integration Program," CLNS-80/447.

[54] J. Prades, "The standard model prediction for muon g-2," arXiv:hep-ph/0108192.

[55] S. Laporta, Int. J. Mod. Phys. A15, 5087 (2000).

[56] M. Hayakawa and T. Kinoshita, arXiv:hep-ph/0112102. 